\title{
Theory of Dissociative Ionization Processes
}

\section{S. KANFER and M. SHAPIRO}

Department of Chemical Physics, Weizmann Institute of Science, Rehovot 76100, Israel

A quantum mechanical theory for the $\hbar \omega+A B \rightarrow A+B^{+}+e^{-}$three body breakup is presented. The theory is based on the Continuum Coupled Channels expansion (CCC) in which the three body wave-function is expanded in terms of square integrable and (flux carrying) continuum states. The theory consistently treats the accompanying processes of three body inelastic collisions, dissociative attachment and Penning ionization. Applications to the dissociative photoionization of $\mathrm{H}_{2}$ and $\mathrm{HD}$ are presented. The relevant equations are solved exactly using the Artificial Channel method. Excellent agreement is obtained with experiment, concerning the proton kinetic energy distribution.

\section{INTRODUCTION}

When molecular hydrogen absorbs a photon with energy above the dissociative ionization threshold, four distinct outcomes are energetically possible:

$$
\begin{array}{ll}
\mathrm{H}_{2} \rightarrow \mathrm{H}_{2}^{+}+e^{-}(\varepsilon) & \text { Ionization } \\
\mathrm{H}_{2} \rightarrow \mathrm{H}(1 s)\left(E_{p}\right)+\mathrm{H}^{+}\left(E_{p}\right)+e^{-}(\varepsilon) & \text { Dissociative ionization } \\
\mathrm{H}_{2} \rightarrow \mathrm{H}(1 s)+\mathrm{H}(n s) n>1 & \text { Neutral dissociation } \\
\mathrm{H}_{2} \rightarrow \mathrm{H}^{+}+\mathrm{H}^{-} & \text {Ion pair formation }
\end{array}
$$

(Here $E_{p}$ denotes the kinetic energy of either a hydrogen atom or proton, and $\varepsilon$ the kinetic energy of an electron.) Indeed there is experimental evidence for the participation of the first two of these channels above the $\mathrm{H}_{2}$ three body threshold $E_{t h}=18.1 \mathrm{eV}^{1-4}$ 
In view of these possibilities, a theoretical analysis of the fate of molecular hydrogen excited at these energies must consider interactions between varieties of two body and three body processes such as

$$
\begin{aligned}
\mathrm{H}(1 s)+\mathrm{H}(n s) & \rightarrow \mathrm{H}^{+}+\mathrm{H}(1 s)+e^{-} \\
\text {Penning ionization } & \\
\mathrm{H}_{2}^{+}+e^{-} \rightarrow \mathrm{H}(1 s)+\mathrm{H}(n s) & \text { Dissociative attachment } \\
\mathrm{H}(1 s)+\mathrm{H}^{+}+e^{-}(\varepsilon) \rightarrow \mathrm{H}(1 s)+\mathrm{H}^{+}+e^{-}\left(\varepsilon^{\prime}\right) & \text { Three-body inelastic scattering }
\end{aligned}
$$

in the course of assigning a probability to an individual dissociation channel. These subtleties were confronted in a preliminary study of the dissociative ionization of $\mathrm{H}_{2}$ out of which emerged the theory of continuum coupled channels. ${ }^{5}$ The theory was found to account successfully for the distribution of proton kinetic energies measured for the three body breakup of $\mathrm{H}_{2}$ at $26.9 \mathrm{eV}$. Here we consider some of the salient features of the continuum coupled channels method, and explore further its application to the dissociative photoionization of $\mathrm{H}_{2}$ and $\mathrm{HD}$.

\section{THEORY}

In the center of mass coordinate system (whose origin is fixed to good approximation along the $\mathrm{H}_{2}$ or $\mathrm{HD}$ internuclear distance) we write the total radiation-matter Hamiltonian as

$$
\mathrm{H}\left(\mathbf{q}_{1} \mathbf{q}_{2} \mid R\right)=T(R)+\mathrm{H}^{\mathrm{el}}\left(\mathbf{q}_{1} \mathbf{q}_{2} \mid R\right)+\mathrm{H}^{\mathrm{rad}}(\omega)+\boldsymbol{\mu}\left(\mathbf{q}_{1} \mathbf{q}_{2} \mid R\right) \cdot \mathbf{F}
$$

where $q_{1}$ and $q_{2}$ are the electronic coordinates $R$ is the $\mathrm{H}-\mathrm{H}$ internuclear separation, $T(R)$ is the nuclear kinetic energy term, $\mathrm{H}^{\mathrm{el}}\left(\mathbf{q}_{1} \mathbf{q}_{2} \mid \boldsymbol{R}\right)$ is the electronic Hamiltonian, given in a.u. as,

$$
\mathrm{H}^{\mathrm{el}}\left(\mathbf{q}_{1} \mathbf{q}_{2}\right)=-\frac{1}{2} \nabla_{1}^{2}-\frac{1}{2} \nabla_{2}^{2}-\frac{1}{q_{1 \mathrm{~A}}}-\frac{1}{q_{1 B}}-\frac{1}{q_{2 A}}-\frac{1}{q_{2 B}}+\frac{1}{q_{12}}+\frac{1}{R}
$$

where $q_{1 A}, q_{1 B}, \ldots$ are distances of electron 1 from nucleus $A$ etc. $\mathrm{H}^{\mathrm{rad}}(\omega)$ is the radiation Hamiltonian for incident photons of frequency $\omega$ and $\boldsymbol{\mu} \cdot \mathbf{F}$ is the dipolar radiation-matter interaction. 
We consider three types of electronic states: (1) The exact ground $\left(X^{1} \Sigma_{g}^{+}\right)$state defined via the equation

$$
\left[V_{\mathrm{g}}(R)-\mathrm{H}^{\mathrm{el}}\left(\mathbf{q}_{1} \mathbf{q}_{2} \mid R\right)\right] \phi_{\mathrm{g}}\left(\mathbf{q}_{1} \mathbf{q}_{2} \mid R\right)=0
$$

(2) An approximate $\left({ }^{1} \Sigma_{u}^{+}\right)$"resonant" state $\phi_{r}\left(\mathbf{q}_{1}, \mathbf{q}_{2} \mid R\right)$ and (3) the host of continuum states $-\phi_{\varepsilon}\left(\mathbf{q}_{1} \mathbf{q}_{2} \mid R\right)$, composed of the ground $\mathrm{H}_{2}^{+}\left({ }^{2} \Sigma_{\mathrm{g}}^{+}\right)$molecule and an electron scattered in the $\mathrm{H}_{2}^{+}$field. $\phi_{r}\left(\mathbf{q}_{1}, \mathbf{q}_{2} \mid R\right)$ is considered approximate because it diagonalizes only part of $\mathrm{H}^{\mathrm{el}}\left(\mathbf{q}_{1}, \mathbf{q}_{2} \mid R\right)$, namely that part which does not autoionize to the $\phi_{\varepsilon}\left(\mathbf{q}_{1}, \mathbf{q}_{2} \mid R\right)$ states. Similarly the $\phi_{\varepsilon}\left(\mathbf{q}_{1} \mathbf{q}_{2} \mid R\right)$ basis functions do not allow for binding of the electron. The continuum states are, in the simplest approximation, represented as,

$$
\begin{aligned}
\phi_{\varepsilon}\left(\mathbf{q}_{1} \mathbf{q}_{2} \mid R\right)= & \frac{1}{2}\left[\phi_{I}\left(\mathbf{q}_{1} \mid R\right) \chi_{\varepsilon, l}\left(\mathbf{q}_{2}\right)+\phi_{I}\left(\mathbf{q}_{2} \mid R\right) \chi_{\varepsilon, l}\left(\mathbf{q}_{1}\right)\right] \\
& \times[\alpha(1) \beta(2)-\beta(1) \alpha(2)]
\end{aligned}
$$

where $\alpha$ and $\beta$ are spin $\frac{1}{2}$ functions, $\phi_{I}\left(\mathbf{q}_{1} \mid R\right)$ is the ground $\mathrm{H}_{2}^{+}$ electronic state,

$$
\left[E_{t h}+V_{I}(R)+\frac{1}{2} \nabla_{1}^{2}+\frac{1}{q_{1 A}}+\frac{1}{q_{1 B}}-\frac{1}{R}\right] \phi_{I}\left(\mathbf{q}_{1} \mid R\right)=0
$$

where $V_{I}(R)$ is the $R$-dependent total electronic energy, $E_{t h}$ is the threshold energy for $\mathrm{H}+\mathrm{H}^{+}$production. $\chi_{\varepsilon, l}\left(\mathbf{q}_{2}\right)$ is an $l$-wave ( $p$-wave in the present case) coulomb wavefunction

$$
\left[\varepsilon+\frac{1}{2} \nabla_{2}^{2}+1 /\left|\mathbf{q}_{2}\right|\right] \chi_{\varepsilon, l}\left(\mathbf{q}_{2}\right)=0
$$

normalized to $\delta\left(\varepsilon-\varepsilon^{\prime}\right)$ function in energy

$$
\left\langle\chi_{\varepsilon} \mid \chi_{\varepsilon^{\prime}}\right\rangle=\delta\left(\varepsilon-\varepsilon^{\prime}\right)
$$

In Eq. (6) $\left|\mathbf{q}_{2}\right|$ is the distance of electron (2) from the $\mathrm{H}_{2}$ center of mass. The diagonal matrix elements of $\mathrm{H}^{\mathrm{el}}$ for these electronic wavefunctions are plotted in Figure 1.

The total (nuclear + electronic) wavefunction $\Psi\left(\mathbf{q}_{1} \mathbf{q}_{2}, R\right)$ which is an eigenfunction of $\mathrm{H}$,

$$
\left[E-\mathrm{H}\left(\mathbf{q}_{1} \mathbf{q}_{2}, R\right)\right] \Psi\left(\mathbf{q}_{1} \mathbf{q}_{2}, R\right)=0
$$




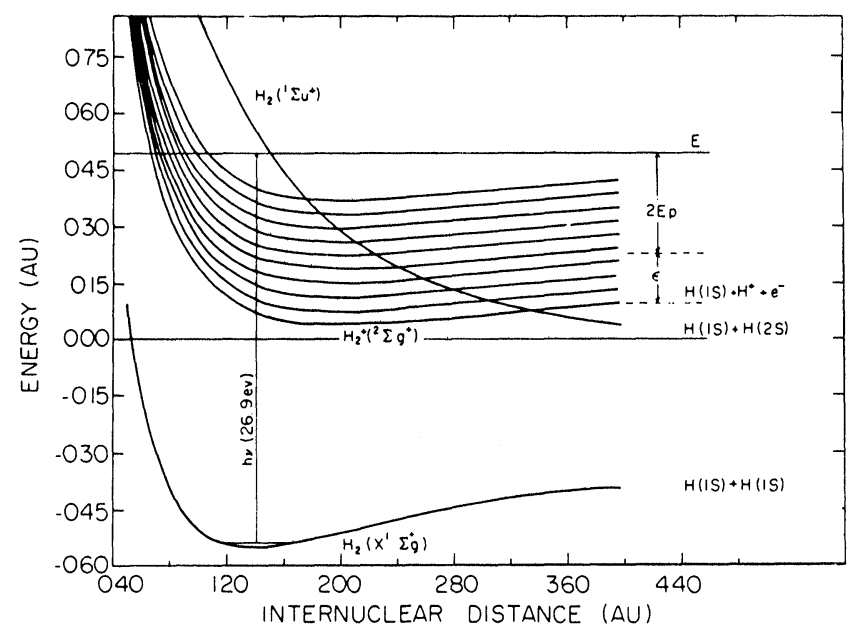

FIGURE 1 Diagonal matrix elements of the electronic Hamiltonian $\mathrm{H}^{\mathrm{el}}$, illustrating the partition of the electronic kinetic energy continuum $\left(E-E_{t h}\right)$ into ten discrete channels. The proton kinetic energy is inferred from the electronic kinetic energy by conservation of energy.

is now expanded as follows,

$$
\begin{aligned}
\Psi\left(\mathbf{q}_{1} \mathbf{q}_{2}, R\right)= & |n\rangle \phi_{\mathrm{g}}\left(\mathbf{q}_{1} \mathbf{q}_{2} \mid R\right) \psi_{\mathrm{g}}(\boldsymbol{R})+|n-1\rangle \phi_{r}\left(\mathbf{q}_{1} \mathbf{q}_{2} \mid R\right) \psi_{r}(\boldsymbol{R}) \\
& +|n-1\rangle \int_{0}^{\varepsilon_{\max }} d \varepsilon^{\prime} \phi_{\varepsilon^{\prime}}\left(\mathbf{q}_{1} \mathbf{q}_{2} \mid R\right) \psi_{\varepsilon}(\boldsymbol{R})
\end{aligned}
$$

where $|n\rangle$ are $n$ photon eigenstates satisfying

$$
\left(H_{\mathrm{rad}}(\omega)-n \hbar \omega\right)|n\rangle=0
$$

and $\varepsilon_{\max }=E-E_{t h}$. We now substitute expansion (8) into Eq. (7) premultiply and integrate by $|n\rangle \phi_{g}$, and $|n-1\rangle \phi_{r}$ in turn. Using the orthonormality of $\phi_{\mathrm{g}}$ and $\phi_{\mathrm{r}}$ we obtain for the discrete components of the wavefunction the following equations,

$$
\begin{gathered}
{\left[E-n \hbar \omega-V_{g}(R)-T(R)\right] \psi_{g}(R)=0} \\
{\left[E-(n-1) \hbar \omega-V_{r}(R)-T(R)\right] \psi_{r}(R)} \\
=F \mu_{r g}(R) \psi_{g}(R)+\int d \varepsilon V_{r \varepsilon}(R) \psi_{\varepsilon}(R)
\end{gathered}
$$


where

$$
\begin{aligned}
V_{r}(R) & \equiv\left(\phi_{r}\left|\mathrm{H}^{\mathrm{el}}\right| \phi_{r}\right) \\
V_{r \varepsilon}(R) & \equiv\left(\phi_{r}\left|\mathrm{H}^{\mathrm{el}}\right| \phi_{\varepsilon}\right)=V_{\varepsilon r}(R) \\
\mu_{r g}(R) & \equiv\left(\phi_{r}|\boldsymbol{\mu} \cdot \mathbf{F}| \phi_{g}\right)
\end{aligned}
$$

with ( ) denoting integration over $\mathbf{q}_{1}$ and $\mathbf{q}_{2}$ only. In Eq. (10a) we have neglected the back-coupling of the initial state to the host of $|n-1\rangle$ photon states. This neglect is not essential but is completely justified for most radiation fields employed in the photoionization experiments. ${ }^{1-3}$

In extracting the continuum components $\psi_{\varepsilon}$ we note that the continuum-continuum matrix elements assume the form,

$$
\begin{aligned}
\left(\phi_{\varepsilon}\left|\mathrm{H}^{\mathrm{el}}\right| \phi_{\varepsilon^{\prime}}\right)= & \left(\phi_{I} \chi_{\varepsilon, l}\left|-\frac{1}{2} \nabla_{1}^{2}-\frac{1}{q_{1 A}}-\frac{1}{q_{1 B}}+\frac{1}{R}-\frac{1}{2} \nabla_{2}^{2}-\frac{1}{\left|\mathbf{q}_{2}\right|}\right| \phi_{I} \chi_{\varepsilon^{\prime}, l}\right) \\
& +\left(\phi_{I} \chi_{\varepsilon, l}\left|-\frac{1}{q_{2 A}}-\frac{1}{q_{2 B}}+\frac{1}{q_{12}}+\frac{1}{\left|\mathbf{q}_{2}\right|}\right| \phi_{I} \chi_{\varepsilon^{\prime}, l}+\chi_{\varepsilon^{\prime}, l} \phi_{I}\right) \\
= & \left(V_{I}(R)+\varepsilon\right) \delta\left(\varepsilon-\varepsilon^{\prime}\right)+V_{\varepsilon \varepsilon^{\prime}}(R)
\end{aligned}
$$

The short range potential,

$$
V_{\varepsilon \varepsilon^{\prime}}(R)=\left(\phi_{I} \chi_{\varepsilon, l}\left|-\frac{1}{q_{2 A}}-\frac{1}{q_{2 B}}+\frac{1}{q_{12}}+\frac{1}{\left|\mathbf{q}_{2}\right|}\right| \phi_{I} \chi_{\varepsilon^{\prime}, l}+\chi_{\varepsilon^{\prime}, l} \phi_{I}\right)
$$

can be made diagonal if a more exact representation of $\chi_{\varepsilon, l}$ is given. In the present application it is neglected. When we do this and premultiply Eq. (7) by $|n-1\rangle \phi_{\varepsilon}$ with $\Psi$, expanded as in Eq. (8), we obtain for $\psi_{\varepsilon}(\boldsymbol{R})$ an indenumerable set of coupled equations,

$$
\begin{array}{cr}
{\left[E-(n-1) \hbar \omega-E_{t h}-\varepsilon-V_{I}(R)-T(R)\right] \psi_{\varepsilon}(R)=} & F \mu_{\varepsilon g}(R) \psi_{g}(R) \\
\vdots & +V_{\varepsilon r}(R) \psi_{r}(R)
\end{array}
$$

where as in Eq. (11c)

$$
\mu_{\varepsilon g}(R) \equiv\left(\phi_{\varepsilon}|\boldsymbol{\mu} \cdot \mathbf{F}| \phi_{g}\right)
$$


Our goal remains the calculation of absorption cross sections from the ground state to individual exit channels. In the case of transition to $\left|\Psi^{(-r)}\right\rangle$ - a state which evolves to the asymptotic limit of the ${ }^{1} \Sigma_{u}$ resonance state,

$$
\sigma(r \leftarrow g)=\frac{8 \pi^{3} \nu}{3 c}\left|\left\langle\Psi^{(-r)}|\mathbf{F} \cdot \boldsymbol{\mu}| \Psi_{g}\right\rangle\right|^{2}
$$

For absorption to a state $\left|\Psi^{(-\varepsilon)}\right\rangle$ which evolves asymptotically to a three body state with definite electron kinetic energy $\varepsilon$ we seek to determine

$$
\sigma(\varepsilon \leftarrow g)=\frac{8 \pi^{3} \nu}{3 c}\left|\left\langle\Psi^{(-\varepsilon)}|\mathbf{F} \cdot \mu| \Psi_{g}\right\rangle\right|^{2}
$$

In the representation of expansion (8)

$$
\begin{gathered}
\Psi_{\mathrm{g}}=\phi_{\mathrm{g}} \psi_{\mathrm{g}} \\
\Psi^{(-r)}(R, \mathbf{q})=\phi_{r} \psi_{r}^{(-r)}+\int_{0}^{\varepsilon_{\max }} d \varepsilon \phi_{\varepsilon} \psi_{\varepsilon}^{(-r)} \\
\Psi^{(-\varepsilon)}(R, \mathbf{q})=\phi_{r} \psi_{r}^{(-\varepsilon)}+\int_{0}^{\varepsilon_{\max }} d \varepsilon^{\prime} \phi_{\varepsilon} \psi_{\varepsilon^{\prime}}^{(-\varepsilon)}
\end{gathered}
$$

and the superscript (-) refers to outgoing spherical wave boundary conditions in the internuclear coordinate $R$. The total dissociative photoionization cross section $\sigma_{\text {dion }}(\{\varepsilon\} \leftarrow g)$ is given by the sum of $\sigma(\varepsilon \leftarrow g)$

$$
\sigma_{\text {dion }}(E)=\int_{0}^{\varepsilon_{\max }} d \varepsilon \sigma(\varepsilon \leftarrow g)
$$

Bound-continuum matrix elements of the form appearing in Eq. (14), (15) are obtained directly using the "artificial channel method,", and to this end we discretize the indenumerable set of equations (10c). This is accomplished by replacing the integral in $(10 \mathrm{~b})$ by a quadrature:

$$
\int_{0}^{\varepsilon_{\max }} d \varepsilon V_{r \varepsilon} \psi_{\varepsilon} \cong \sum_{i=1}^{N} \Delta \varepsilon_{i} V_{r \varepsilon_{i}} \psi_{\varepsilon_{i}}
$$

where $\Delta \varepsilon_{i}$ are products of quadrature weights (repeated midpoint ${ }^{9}$ ) and quadrature intervals. Our criteria of convergence are the overall shape of the distribution of partial absorptions, and total photoioni- 
zation cross section, now represented by a quadrature sum:

$$
\sigma_{\text {dion }}(E)=\sum_{i=1}^{N} \Delta \varepsilon_{i} \sigma\left(\varepsilon_{i} \leftarrow g\right)
$$

\section{RESULTS AND DISCUSSION}

\section{1. $\mathrm{H}_{2}$}

The distribution of proton kinetic energies calculated for photon energy of $26.9 \mathrm{eV}$ and $N=67$ is shown in Figure 2, together with the experimental data of Browning. ${ }^{3}$ Both low and high proton kinetic energy regions are accurately reproduced. Figure 3 shows more clearly the quality of agreement between theory and experiment in the region of high proton kinetic energies. For $65 \leqslant N \leqslant 67$, integration of the calculated distribution gives $\sigma_{\text {dion }}=1.41 \times 10^{-3} a_{0}^{2}( \pm 0.19 \%)$. This is in excellent agreement with both experimental ${ }^{1,10}$ and theoretical ${ }^{10,11}$ values for this cross section, all in the neighborhood of $2 \times 10^{-3} a_{0}^{2}$. The quadrature points in this calculation could be chosen with either

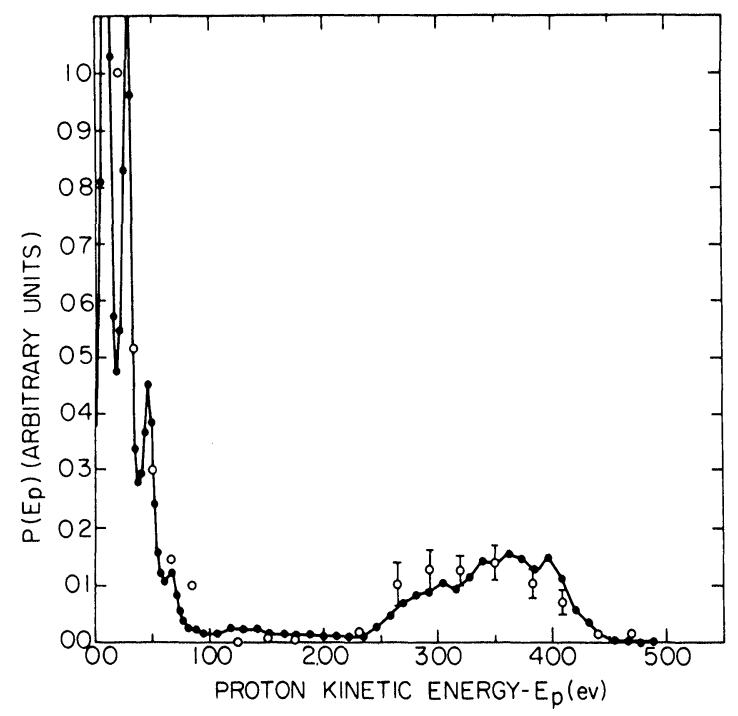

FIGURE 2 Experimental (-O-) proton kinetic energy distribution from $\mathrm{H}_{2}$ at photon energy of $26.9 \mathrm{eV}$ (see Ref. 3), and calculated (- - distribution. 


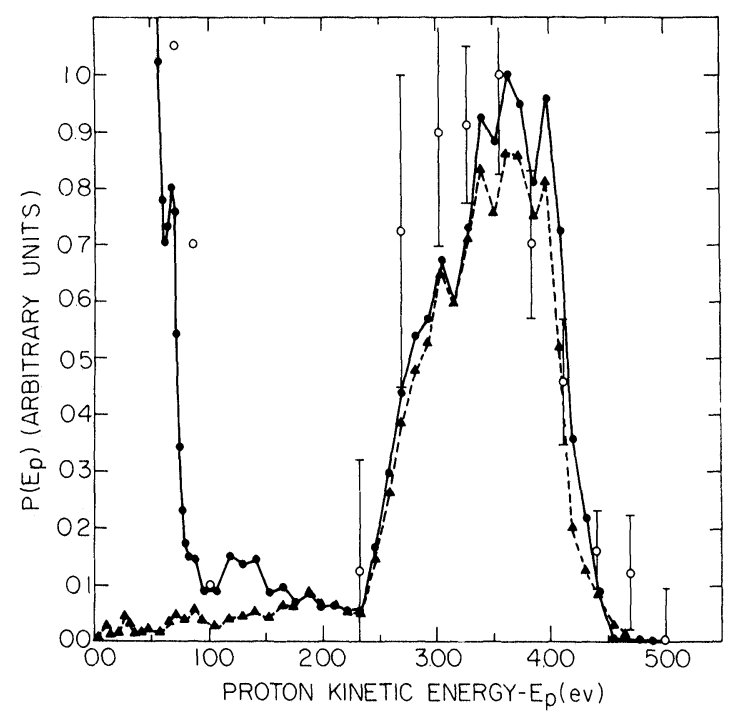

FIGURE 3 Experimental (-O-) proton kinetic energy distribution, and calculated distributions. (-O) $\mu_{e g}, V_{r \varepsilon}$ included (as in Figure 2), (-- $\left.-\Delta--\right) \mu_{g \mathrm{gg}}$ neglected.

uniform or varying density across the range of available energy without affecting the shape of the distribution or the integrated cross section.

Again for $65 \leqslant N \leqslant 67$, integration of the high proton kinetic peak alone affords $\sigma_{\text {dion }}\left(E_{p}>2 \mathrm{eV}\right)=4.2 \times 10^{-4} a_{0}^{2}( \pm 0.003 \%)$. This is in reaonable agreement with the only experimental estimate of this cross section in the literature: $2.5 \times 10^{-4} a_{0}^{2}<\sigma\left(E_{p}>2 \mathrm{eV}\right)<5.4 \times 10^{-4} a_{0}^{2}$ and serves as the basis for the scaling of the experimental and theoretical distributions in Figures 2 and 3.

The role of direct $\mu_{\varepsilon g}$ and autoionization $V_{r e}$ processes in the production of protons with low and high kinetic energies was deduced by alternatively suppressing $\mu_{e g}$ and $V_{r \varepsilon}$ in the calculation. When $V_{r \varepsilon}$ is suppressed, the high proton kinetic energy peak disappears and the low energy peak remains, although reduced in intensity by $7 \%$. Similarly, when $\mu_{\varepsilon g}$ is suppressed the dashed line spectrum of Figure 3 is obtained: here the low energy peak is absent, and the high energy peak is reduced by $13 \%$ from Figure 2 . All compound processes which originate in direct absorption to the continuum, undergo dissociative attachment and secondary autoionization are represented 
in this factor of $13 \%$. Most significantly, these compound processes preserve the kinetic energy distribution characteristic of the final ionization.

The distinct low and high energy features of Figures 2 and 3 appear when we demand that $V_{r \varepsilon_{i}}=0$ for $R>R_{\varepsilon_{i}}^{c}$ where $R_{\varepsilon_{i}}^{c}$ is the internuclear distance where the resonant state and continuum channel potentials cross. In the Born-Oppenheimer approximation this is rigorously true, for at distances $R_{\varepsilon_{i}}^{c}+\delta R$ where $\delta R>0$, the nuclei moving under the potential $V_{r}(R)$ have acquired more kinetic energy than will permit production of an electron with energy $\varepsilon_{i}$ and conserve total energy. The absolute magnitudes of the nonzero elements of $V_{r e}$ have been reduced by a factor of 0.35 so as to afford the quantitative agreement with the experimental cross sections of Figures 2 and 3.

\section{HD}

The complete treatment of the dissociative photoionization of HD must account for the possibility of $\left\{\mathrm{H}^{+}+\mathrm{D}(1 s)\right\}$ as well as $\left\{\mathrm{D}^{+}+\mathrm{H}(1 s)\right\}$

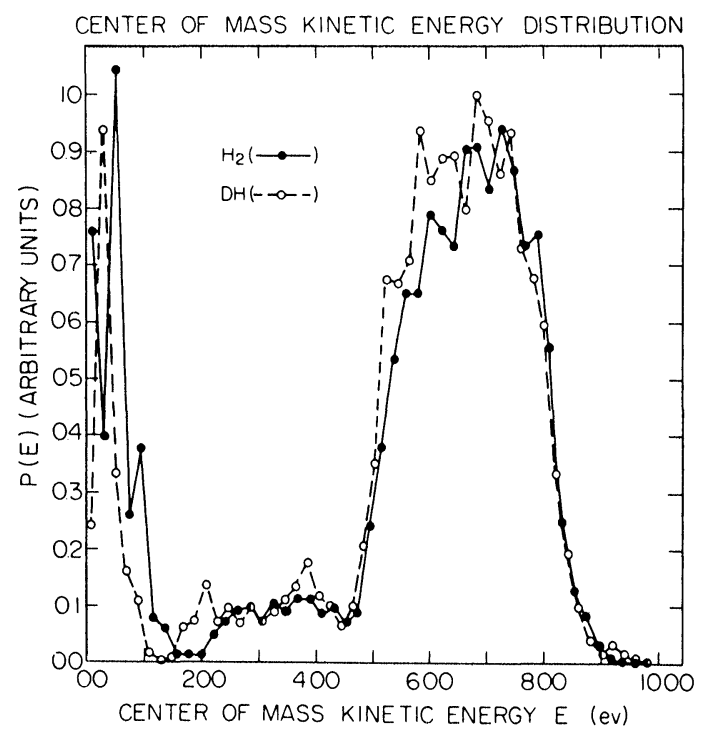

FIGURE 4 Center of mass kinetic energy distribution for $\mathrm{H}_{2}(--)$ and $\mathrm{HD}$ $\left(---\mathrm{O}_{---)}\right.$at $26.9 \mathrm{eV}$. 
pairs and, consequently, include both ground and excited $\phi_{\varepsilon}^{*}$ basis functions in expansion (8). This, in turn, will produce ground and excited $V_{I}^{*}$ potential functions in Eqs. (10c). Some experimental $\mathrm{H}^{-} / \mathrm{HD}^{+}$and $\mathrm{D}^{-} / \mathrm{HD}^{+}$ratios of order unity have been reported for lower energy photons, ${ }^{12}$ and we shall assume here that the $\mathrm{H}_{2}$ coupling scheme presented above is appropriate for the dissociative photoionization of $\mathrm{HD}$, with $\left(\mathrm{H}^{+}\right) /\left(\mathrm{D}^{+}\right)=1$.

Model calculations have been carried out on $\mathrm{H}_{2}$ and $\mathrm{HD}$ at $26.9 \mathrm{eV}$ with coupling potentials $V_{r \varepsilon}$ and $\mu_{\varepsilon g}$ which produce low and high energy peaks of similar intensity. The center of mass kinetic energy distributions shown in Figure 4 reveal a shift to lower energies of both the high energy and low energy peaks on passage from $\mathrm{H}_{2}$ to HD. This shift to lower energies is consistent with the behavior of both direct and resonance processes of $\mathrm{H}_{2}$ when the photon frequency is decreased: ${ }^{3}$ in our case, the shift of photon frequency is represented by the shift in zero point vibrational energy on going from $\mathrm{H}_{2}$ to $\mathrm{HD}$ $(0.0729 \mathrm{eV})$. From the center of mass kinetic energy distribution of

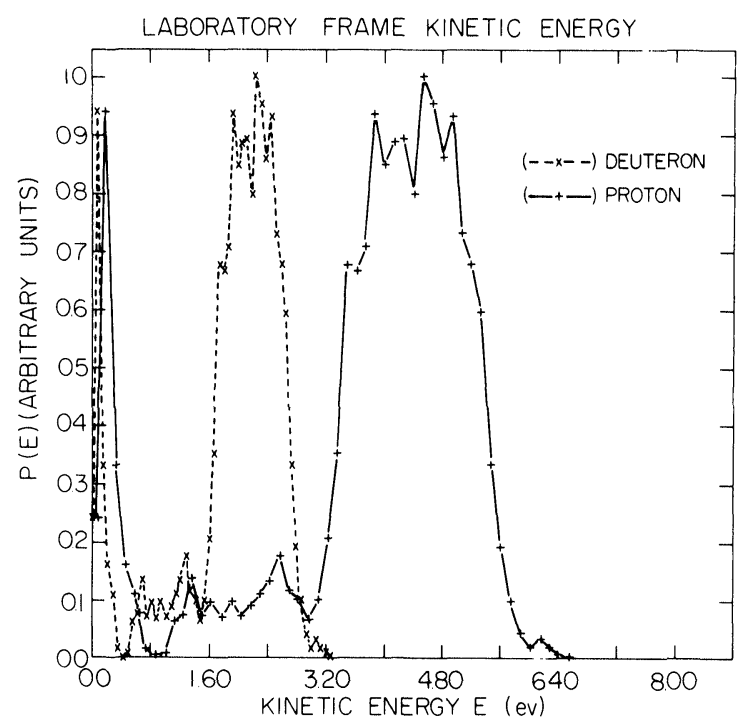

FIGURE 5 Laboratory frame kinetic energy distributions for $\mathrm{HD}$ fragments at $26.9 \mathrm{eV}$, derived from Figure 4 , using the relations $E_{\mathrm{D}^{+}}=(1 / 3) \times E_{\mathrm{C} . \mathrm{M}}, E_{\mathrm{H}^{+}}=$ $(2 / 3) \times E_{\text {C.M. }}$ and $\left(\mathrm{H}^{+}\right) /\left(\mathrm{D}^{+}\right)=1 .(---\times---) \mathrm{D}^{+},(-+-) \mathrm{H}^{+}$. 
Figure 4 and an assumed branching ratio of unity we calculate the laboratory frame kinetic energy distribution for $\mathrm{H}^{+}$and $\mathrm{D}^{+}$shown in Figure 5.

The proper treatment of closed continuum channels, i.e., that part of the integral in Eq. (10b) with limits $(E, \infty)$, as well as the related problem of formation of bound $\mathrm{H}_{2}^{+}$, have not yet been discussed in the context of the continuum coupled channels method, and are presently under investigation. A rigorous proof for the existence of convergent cross sections based on continuum Coulomb wavefunctions is also being sought. The full scope of the continuum coupled channels method will emerge as these questions are clarified.

\section{References}

1. R. Browning and J. Fryar, J. Phys. B 6, 364 (1973).

2. S. Strathdee and R. Browning, J. Phys. B 9, L505 (1976).

3. S. Strathdee and R. Browning, J. Phys. B 12, 1789 (1979).

4. J. E. Pollard, D. J. Trevor, J. E. Reutt, Y. T. Lee and D. A. Shirley, J. Chem. Phys. 77, 34 (1982).

5. S. Kanfer and M. Shapiro, submitted for publication.

6. A. L. Ford, K. K. Docken and A. Dalgarno, Astrophys. J. 195, 819 (1975).

7. K. Kirby, S. Guberman and A. Dalgarno, J. Chem. Phys. 70, 4635 (1979).

8. M. Shapiro, J. Chem. Phys. 56, 2582 (1972).

9. F. B. Hildebrand, Introduction to Numerical Analysis, 2nd ed. (McGraw-Hill Inc., New York, 1974).

10. S. V. O'Neil and W. P. Reinhardt, J. Chem. Phys. 69, 2126 (1978) and experimental references therein.

11. K. Kirby, T. Uzer, A. C. Allison and A. Dalgarno, J. Chem. Phys. 75, 2820 (1981).

12. W. A. Chupka, F. M. Dehmer and W. T. Jivery, J. Chem. Phys. 63, 3929 (1975). 